\title{
Conhecimento e percepção de risco sobre o HIV/AIDS: um perfil da população brasileira no ano de 1998
}

\author{
Knowledge and risk perception of HIV/AIDS: \\ a profile of the Brazilian people in 1998
}

\footnotetext{
1 Fundação Sistema Estadual de Análise de Dados. Av. Casper Líbero 464, São Paulo, SP 01033-000, Brasil. mpferrei@seade.gov.br
}

Abstract This paper focuses on cognitive aspects related to HIV/AIDS by presenting distinct subgroups of the Brazilian population. Four subgroups were created according to their different levels of knowledge regarding HIVIAIDS and risk perception: subgroup 1 (no awareness and no risk perception); subgroup 2 (no awareness, with some risk perception); subgroup 3 (some awareness and no risk perception); and subgroup 4 (some awareness and some risk perception). The data come from the National Survey on Sexual Behavior and HIV/AIDS Risk Perception in the Brazilian Population, conducted by CEBRAP (the Brazilian Analysis and Planning Agency) together with the Ministry of Health, in 1998. The study highlights that in 1998, slightly over half of the Brazilian population were minimally aware of HIV transmission processes. Analysis of demographic and socioeconomic characteristics associated with protective behavior against HIVIAIDS showed that approximately 23\% of the study population belonged to subgroup 1 with no awareness and no risk perception - and were thus highly vulnerable to infection.

Key words HIV; Acquired Immunodeficiency Syndrome; Risk

Resumo Com o intuito de abordar os aspectos cognitivos sobre o HIVIAIDS, o presente trabalho apresenta subgrupos populacionais distintos, segundo nivel de conhecimento em relação ao HIVIAIDS e percepção de risco. Foram gerados quatro subgrupos: grupo 1 (sem informação e sem percepção de risco), grupo 2 (sem informação e com alguma percepção de risco), grupo 3 (com informação e sem percepção de risco) e grupo 4 (com informação e alguma percepção de risco). Os dados utilizados são referentes à Pesquisa Nacional sobre Comportamento Sexual da População Brasileira e Percepções do HIV/AIDS, realizada em 1998 pelo Centro Brasileiro de Análise e Planejamento e Ministério da Saúde. Entre os resultados obtidos no estudo destaca-se o fato de que pouco mais da metade da população brasileira estava minimamente informada a respeito das formas de transmissão do vírus HIV no ano da pesquisa. Observou-se ainda, que aproximadamente 23\% da população investigada pertencia ao grupo 1 - sem informação e sem percepção de risco - estando portanto, vulneráveis ao HIV quanto à sua condição de compreender seu próprio risco de exposição à AIDS.

Palavras-chave HIV; Síndrome de Imunodeficiência Adquirida; Risco 


\section{Introdução}

A pandemia da infecção pelo vírus HIV constitui-se em um fenômeno, cuja forma de ocorrência, nas diferentes partes do mundo, configura-se como epidemias regionais com características e determinantes próprios.

No Brasil, a epidemia revela múltiplas dimensões, refletindo as profundas desigualdades sociais, econômicas e demográficas da sociedade brasileira. Para Parker \& Camargo Jr. (2000), em um país como o Brasil, de dimensões continentais e importantes disparidades sociais, é de se esperar que esses fatores se reflitam também na forma como o HIV se propaga. Para eles, "estas diferentes características conformariam populações diferentes, ainda que em um mesmo território, com variações quanto à probabilidade de que seus componentes viessem a infectar-se pelo HIV, configurando, portanto, diferentes 'vulnerabilidades' à infecção pelo HIV e, por conseguinte, à AIDS" (Parker \& Camargo Jr., 2000:90).

Ao longo dos anos, o perfil epidemiológico da infecção no país sofreu importantes transformações, de tal forma que a circunscrição da incidência dos casos nos grandes centros urbanos entre homens, prioritariamente com prática homossexual ou hemofílicos, alterou-se paulatinamente para uma maior abrangência geográfica da infecção, atingindo as regiões menos urbanizadas e populosas do Brasil, e parcelas crescentes de mulheres entre os casos notificados. Como conseqüência, a epidemia mostra hoje um "quadro marcado pelos processos de heterossexualização, da feminização, da interiorização e da pauperização" (Brito et al., 2000: 208).

Por meio desse quadro é possível afirmar que a AIDS se propaga entre os grupos populacionais em situações mais vulneráveis, tanto na escala sócio-econômica quanto em questões de gênero. A isso une-se a precária situação social e econômica de grande parte da população brasileira, fazendo com que o acesso à informação e aos serviços de saúde se torne extremamente difícil, quando não impossível. Para as mulheres, a situação pode ser mais grave, pois freqüentemente sofrem as conseqüências tanto da desigualdade sócio-econômica quanto da de gênero (Bastos \& Szwarcwald, 2000).

Para Parker \& Camargo Jr. (2000), no Brasil estão presentes todos os diferentes fatores sócio-econômicos identificados como estruturantes da vulnerabilidade relacionada à AIDS. Além disso, tem-se como agravantes a baixa incorporação dos valores da cidadania e a falta de um sistema de bem-estar social inclusivo que, caso presentes, seriam potencialmente mitigadores dos efeitos dos fatores sócio-econômicos estruturais da vulnerabilidade.

Ayres et al. (1999:57) definem a vulnerabilidade individual com base em três pressupostos: "1) Todo indivíduo é, em algum grau, vulnerável à infecção pelo HIV e suas conseqüências, e essa vulnerabilidade pode variar ao longo do tempo em função dos valores e recursos que lhe permitam ou não obter meios para se proteger; 2) Os indivíduos infectados pelo HIV têm seu potencial de vulnerabilidade a morbidade, invalidez ou morte, variável em função inversa ao amparo social e assistência à saúde de que dispuserem; 3) As condições que afetam a vulnerabilidade individual são de ordem cognitiva (informação, consciência do problema e das formas de enfrentá-lo), comportamentais (interesse e habilidade para transformar atitudes e ações a partir daqueles elementos cognitivos) e sociais (acesso a recursos e poder para adotar comportamentos protetores)".

Nesse contexto, apenas informações sobre formas de transmissão e situações de risco podem ser insuficientes para a adoção de comportamentos protetores. Ou seja, a transformação do conhecimento na adoção de práticas protetoras é mediada por questões de gênero, classe social, etnia e outros componentes sociais que não se localizam apenas na esfera individual. Assim, o poder do conhecimento na troca do comportamento depende das alternativas e perspectivas existentes para o indivíduo (André, 1999; Paicheler, 1999).

Em tal perspectiva, os indivíduos irão construir suas próprias definições de risco de transmissão do HIV, por meio da via sexual, utilizando parte do discurso epidemiológico e preventivo, mas principalmente de acordo com sua posição social, identidade pessoal e tipo de relação na qual está envolvido. Esse foi o caso dos homossexuais dos países desenvolvidos, onde a mudança no comportamento não foi um processo em que indivíduos receberam informações e, valendo-se delas, tomaram decisões racionais sobre seu comportamento. Mas, sim, um processo em que a mudança de comportamento se deu como resultado de alterações nos padrões comportamentais da comunidade em que se inseriam, e com a qual se identificavam (Carr, 1991).

Assim, apesar de se ter ciência de que, no caso da AIDS, o grau de informação que um indivíduo possui sobre formas de transmissão e situações de risco não é suficiente para que passe a adotar um comportamento protetor, a falta de informações básicas contribui para aumentar a sua vulnerabilidade (Peruga \& Celentano, 1993). 
$\mathrm{Na}$ ótica das políticas públicas para o combate ao HIV/AIDS é imprescindível conhecer o nível de informação e a percepção que a população possui sobre o assunto, dispor de informações sobre seu comportamento sexual, além de conhecer as suas condições de vida.

A Pesquisa Nacional sobre Comportamento Sexual da População Brasileira e Percepções do HIVIAIDS, realizada em 1998 pelo Centro Brasileiro de Análise e Planejamento (CEBRAP) e Ministério da Saúde, apresentou entre seus principais resultados que, de nove questões investigadas sobre formas de transmissão e situações de risco associadas ao vírus HIV, aproximadamente $40 \%$ da população investigada não responderam corretamente nem a seis questões, e $8 \%$ (4,8 milhões de pessoas) não responderam corretamente nem a três das nove questões consideradas. As questões referiam-se ao uso de camisinha masculina ou feminina, contato social com portadores do vírus HIV ou doentes de AIDS, compartilhamento de agulhas e seringas, sexo oral, coito interrompido, relações monogâmicas, não-monogâmicas e múltiplas parcerias.

Da população investigada, $3 \%$ mostraramse totalmente informada sobre o HIV/AIDS, acertando todas as nove questões investigadas, o que corresponde a cerca de 1,8 milhão de pessoas. A esse resultado soma-se o fato que até mesmo os indivíduos que responderam corretamente a 7 ou 8 questões, do total das nove investigadas, possuíam dúvidas básicas para a prevenção do HIV/AIDS, tais como a transmissão por meio do coito interrompido ou uso de seringas e agulhas já utilizadas.

Ao se considerar ainda que apenas $4,3 \%$ das pessoas entrevistadas consideravam-se suficientemente esclarecidas, que 5,3\% declararam não ter aprendido como se proteger da AIDS e que 9,6\% alegaram ter aprendido sozinhas, pode-se inferir que ainda existe uma parcela significativa da população que não se mostra totalmente informada sobre AIDS (CEBRAP, 1999).

Com o intuito de abordar, ainda que parcialmente, os aspectos cognitivos sobre o HIV/ AIDS e contribuir para a discussão metodológica de indicadores para a formulação de políticas públicas em saúde, o presente estudo apresenta um indicador de conhecimento em relação ao HIV/AIDS e subgrupos populacionais distintos, segundo níveis de conhecimento e percepção de risco.

\section{Metodologia}

Os dados utilizados no trabalho são referentes à Pesquisa Nacional sobre Comportamento Sexual da População Brasileira e Percepções do HIV/AIDS, realizada em 24 Estados brasileiros e o Distrito Federal. Tal levantamento teve como população alvo os moradores das áreas urbanas de 183 microrregiões, com idade entre 16 e 65 anos, abrangendo aproximadamente $76 \%$ da população brasileira nesta faixa etária, residente em áreas urbanas do país. A pesquisa investigou o nível de conhecimento da população brasileira sobre AIDS e demais doenças sexualmente transmissíveis, comportamento sexual, saúde reprodutiva e questões referentes às características sócio-econômicas e demográficas (CEBRAP, 1999).

A coleta de informações baseou-se em uma amostra probabilística em múltiplos estágios, com 3.600 domicílios espalhados por $168 \mathrm{mu}$ nicípios brasileiros. A unidade de primeiro estágio foi a microrregião; a de segundo, o setor censitário; a de terceiro, o domicílio particular; e a de quarto estágio, o morador com idade entre 16 e 65 anos (CEBRAP, 1999).

Na composição do indicador de conhecimento, consideraram-se questões referentes às formas de transmissão do vírus e situações de risco. É importante destacar que foram escolhidas as mesmas questões que a Pesquisa $\mathrm{Na}$ cional sobre Comportamento Sexual da População Brasileira e Percepções do HIVIAIDS utilizou na construção de um indicador deste tipo. Da mesma forma, o tratamento dado a cada uma das questões também foi análogo ao utilizado pela pesquisa nacional (CEBRAP, 1999).

Com base em questões sobre mecanismos de transmissão do vírus HIV e formas de proteção, os indivíduos foram classificados como bem-informados ou mal-informados em seis itens: uso da camisinha masculina, uso da camisinha feminina, contato social com portadores do vírus HIV ou doentes de AIDS, uso compartilhado de agulhas e seringas, coito interrompido e sexo oral. Para os dois primeiros itens foram considerados bem-informados os que apontaram o uso da camisinha como uma forma de se proteger do HIV/AIDS; na questão referente a contato social com portadores ou doentes de HIV/AIDS foram classificados como bem-informados os que não a consideraram como uma forma de transmissão do vírus; nos três últimos itens a categoria bem-informado engloba os indivíduos que estavam informados de que o vírus pode ser transmitido por meio de tais práticas. Os que não responderam a algumas das questões foram excluí- 
dos, e os que declararam não saber responder à questão foram considerados como mal-informados.

Quanto às situações de risco foram consideradas três: "casais monogâmicos" (heterossexuais ou homossexuais), "casais não-monogâmicos" (heterossexuais ou homossexuais) e "pessoas com muitos parceiros", independentemente do sexo. No caso de casais monogâmicos foram classificados como bem-informados aqueles que não isentaram de risco esta situação; para as duas últimas situações, os bem-informados são aqueles que as consideraram como de alto risco. O tratamento da não resposta foi o mesmo que o realizado para as questões referentes às formas de transmissão.

Os modelos da Teoria da Resposta ao Item (TRI) foram aplicados nos dados. Essa técnica utiliza modelos matemáticos para a construção de indicadores baseados em um conjunto de variáveis observáveis que conjuntamente expressam uma característica latente do indivíduo. Tal procedimento sugere formas de representar a relação entre a probabilidade de um indivíduo responder corretamente a uma questão e seu conhecimento na área analisada. Atualmente, essa técnica é utilizada no Brasil na área de avaliação educacional, na qual são construídas as chamadas escalas de habilidade, que permitem classificar alunos segundo seu desempenho em testes educacionais. (MEC, 1999).

Para cada um dos nove componentes foi ajustado o modelo logístico de Birnbaum. Nesse tipo de modelo considera-se que a probabilidade de um indivíduo responder corretamente a uma determinada questão, dado o seu conhecimento sobre o assunto, possui uma distribuição logística, definida com base em dois parâmetros (Andrade et al., 2000).

A expressão matemática para este modelo é:

$\Psi_{j}(\theta)=\frac{1}{1+e^{-z_{j}}}($ Modelo de Birnbaum)

onde, $\Psi_{j}(\theta)$ corresponde à probabilidade de um indivíduo responder corretamente ao item $j(j=1, \ldots, 9)$, dado o seu conhecimento sobre HIV/AIDS que é denotado por $\theta ; z_{j}=a_{j} \theta+c_{j} ; a_{j}$ : corresponde ao coeficiente angular e está relacionado ao poder de discriminação do item. No presente caso pode ser interpretado como o poder do item ou questão de discriminar o conhecimento de um indivíduo sobre o HIV/ AIDS; $c_{j}$ : corresponde ao intercepto.

A qualidade do ajuste foi avaliada por meio dos resíduos dos modelos e de uma estatística Qui-Quadrado.
Valendo-se desses modelos foi obtido um escore $\theta, \operatorname{com}$ intervalo de variação entre $0 \mathrm{e}$ 100 , correspondendo ao indicador de conhecimento sobre HIV/AIDS. O valor zero corresponde à resposta incorreta em todas as questões investigadas, e o valor 100 , à resposta correta em todas elas. Tal indicador não pode ser expresso explicitamente como uma função dos seus nove componentes, pois é obtido por meio de métodos interativos (Mislevy \& Bock, 1990).

Com os resultados obtidos pelos modelos de TRI é possível identificar, entre os componentes do indicador, aqueles com maiores ou menores graus de dificuldade e discriminação, por intermédio dos índices de dificuldade e de discriminação. O índice de dificuldade corresponde à proporção de pessoas bem-informadas em cada um dos nove componentes considerados: maiores proporções indicam questões mais simples. O índice de discriminação indica quanto uma questão é capaz de diferenciar pessoas com base no conhecimento. Esse valor é calculado pela diferença entre a proporção de pessoas que acertaram a questão nos grupos superior e inferior do indicador. O grupo inferior é composto pelos $30 \%$ dos indivíduos com pior desempenho no indicador, e o grupo superior é composto pelos $30 \%$ dos indivíduos com melhor desempenho no indicador. Quanto maior for o índice de discriminação melhor é a questão para diferenciar o conhecimento sobre o HIV/AIDS (MEC, 1999).

Uma das possibilidades do tipo de escala criado é poder se obter pontos "interpretáveis", que são definidos matematicamente e são chamados níveis âncoras. Um dos níveis encontrados foi o ponto 74 na escala, pois praticamente todas as pessoas que atingem esta pontuação estão bem-informadas sobre o uso da camisinha como forma de proteção, que a transmissão não ocorre por intermédio do contato social com portadores do vírus HIV ou doentes de AIDS, e que o vírus pode ser transmitido por meio do uso compartilhado de agulhas e seringas. Portanto, definiu-se como minimamente informado um indivíduo que atinge 74 pontos na escala de 0 a 100 de conhecimento sobre o HIV/AIDS.

Definiu-se um segundo corte na escala (90 pontos) correspondendo aos indivíduos com um bom nível de informação sobre o HIV/AIDS. O percentual de respostas corretas, entre as pessoas que atingem esta pontuação, para cada um dos componentes do indicador é de no mínimo 65\%.

Para a mensuração da percepção do próprio risco de se contaminar foi utilizada a questão do questionário referente ao risco auto-atribuí- 
do. Foram criadas duas categorias nenhum risco e algum risco, contendo as pessoas que declararam um risco baixo, médio ou alto. Os indivíduos que não souberam responder à questão foram classificados na categoria nenhum risco, e os que não quiseram responder à questão, 0,1\% dos respondentes, não foram considerados na análise.

Com base no cruzamento dos indicadores de conhecimento sobre o HIV/AIDS (minimamente informado e não está informado) e risco auto-atribuído (nenhum risco e algum risco) foram criados quatro subgrupos populacionais:

- Grupo 1 - sem informação e sem percepção de risco: contendo os indivíduos que não possuem o nível mínimo de informação e não possuem a percepção do próprio risco. Este grupo seria o grupo mais vulnerável ao HIV quanto à sua condição de compreender seu próprio risco de exposição à AIDS.

- Grupo 2 - sem informação e com alguma percepção de risco: englobando os indivíduos que apesar de não possuírem um nível de conhecimento mínimo, possuem alguma percepção do próprio risco. Talvez a existência de programas educativos sobre o HIV/AIDS para esse grupo possa facilitar a incorporação de práticas e comportamentos protetores.

- Grupo 3 - com informação e sem percepção de risco: englobando os indivíduos que apesar de possuírem um nível de conhecimento mínimo, não possuem a percepção do próprio risco.
Esse grupo apesar de possuir informação não identifica a AIDS como um risco à sua saúde.

- Grupo 4-com informação e alguma percepção de risco: englobando os indivíduos que possuem um nível de conhecimento mínimo e a percepção do próprio risco. Em tese, esse grupo se encontra mais bem posicionado quanto aos aspectos cognitivos da vulnerabilidade em relação ao HIV/AIDS.

\section{Resultados}

A Tabela 1 apresenta, para cada um dos itens sobre conhecimento, os respectivos índices de dificuldade e de discriminação. É possível observar que as questões com os maiores níveis de dificuldade foram referentes ao risco associado à transmissão da AIDS entre casais monogâmicos $(0,500)$, ao uso da camisinha feminina como fator de proteção ao HIV/AIDS $(0,517)$ e à transmissão do vírus HIV/AIDS por meio do contato social com portadores $(0,545)$. Quanto às questões referentes ao conhecimento em relação ao HIV/AIDS utilizadas no estudo, a informação sobre o uso do preservativo como um mecanismo de proteção foi o fator que mais diferenciou as pessoas quanto ao conhecimento. Em menor escala apareceram as informações sobre contato social e uso compartilhado de agulhas e seringas. As demais questões apresentaram pequeno poder de di-

Tabela 1

Índices de dificuldade e de discriminação do item. Brasil, 1998.

\begin{tabular}{lcc}
\hline $\begin{array}{l}\text { Componentes do indicador } \\
\text { de conhecimento sobre o HIV/AIDS }\end{array}$ & $\begin{array}{l}\text { Índice de } \\
\text { dificuldade }\end{array}$ & $\begin{array}{c}\text { Índice de } \\
\text { discriminação }\end{array}$ \\
\hline Uso da camisinha masculina como forma de proteção & 0,692 & 0,993 \\
Uso da camisinha feminina como forma de proteção & 0,545 & 0,912 \\
Transmissão do vírus HIV por meio de contato social & 0,517 & 0,418 \\
com portadores de HIV ou doentes de AIDS1 & 0,599 & 0,309 \\
Transmissão do vírus HIV por meio do uso & & 0,069 \\
compartilhado de agulhas e seringas & 0,594 & 0,035 \\
Transmissão do vírus HIV por meio do coito interrompido & 0,788 & $-0,101$ \\
Transmissão do vírus HIV por meio de sexo oral & 0,500 & 0,141 \\
Situação de risco: casais monogâmicos2 & 0,808 & 0,081 \\
Situação de risco: casais não-monogâmicos 2 & 0,945 &
\end{tabular}

Fonte: Pesquisa Nacional sobre Comportamento Sexual da População Brasileira

e Percepções do HIV/AIDS (Secretaria de Assistência à Saúde, Ministério da Saúde).

1 Neste item foram considerados "bem-informados" apenas os indivíduos classificados

simultaneamente nesta categoria nas questões "Usando banheiros públicos",

"Comendo no mesmo prato de pessoas com AIDS" e "Tocando em pessoas com AIDS".

2 Inclui casais homossexuais.

3 Inclui parceiros do mesmo sexo. 
ferenciar pessoas quanto ao seu conhecimento em relação à AIDS.

Em relação ao nível de informação sobre o HIV/AIDS, o percentual de pessoas que atingiram um nível mínimo de informação (74 pontos ou mais) foi de $51,7 \%$. Entre as mulheres, $53,8 \%$ atingiu este patamar contra $49,5 \%$ dos homens. As pessoas com um bom nível de informação (90 pontos ou mais) correspondiam a $35,2 \%$ da população, sendo que entre as mulheres este percentual foi de $38,1 \%$ e entre os homens 32,0\% (Tabela 2).

Entre os jovens com idade entre 16 e 24 anos observou-se que $52,4 \%$ possuíam um nível mínimo de informação; o contingente com um bom conhecimento nesta faixa etária foi de $30,5 \%$. As jovens se mostraram mais bem-informadas do que os jovens $-57,1 \%$ contra $47,8 \%$. Resultado análogo foi observado para o segundo ponto de corte do indicador, referente a um bom nível de informação, para o qual 34,5\% das mulheres com idade entre 16 e 24 anos atingiram esta pontuação; entre os homens dessa mesma faixa etária esse percentual foi de apenas 25,8\% (Tabela 2).

Quanto à classificação sócio-econômica, $61,1 \%$ dos indivíduos pertencentes à classe A se mostraram minimamente informados em relação à AIDS e 38,3\% possuíam um bom conhecimento. Na classe E tais percentuais foram, respectivamente, $47,7 \%$ e $19,4 \%$. Das mulheres pertencentes à classe A, $61,2 \%$ possuíam conhecimento mínimo e $46,6 \%$ apresentavam um bom conhecimento. Entre os homens pertencentes a essa mesma classe os percentuais foram, respectivamente, $60,9 \%$ e $32,1 \%$. As pessoas pertencentes à classe $\mathrm{E}$ apresentaram os menores níveis de conhecimento, tanto entre os homens como entre as mulheres (Tabela 2).

Quanto aos agrupamentos gerados, observou-se que, o primeiro grupo, com $23,3 \%$ da população ou cerca de 13,8 milhões de pessoas, é composto por maioria masculina; possui a maior média de idade (39 anos) e o menor contingente de jovens; aproximadamente $64 \%$ dos seus membros são casados. Em contrapar-

Tabela 2

Percentual de pessoas de 16 a 65 anos, por nível de conhecimento sobre o HIV/AIDS, segundo características demográficas e sócio-econômicas. Brasil, 1998.

\begin{tabular}{|c|c|c|}
\hline $\begin{array}{l}\text { Características demográficas } \\
\text { e sócio-econômicas }\end{array}$ & $\begin{array}{l}\text { Possui informação } \\
\text { mínima }(\%)^{2}\end{array}$ & $\begin{array}{l}\text { Possui um bom nível } \\
\text { de informação }(\%)^{3}\end{array}$ \\
\hline Total & 51,7 & 35,2 \\
\hline Mulheres & 53,8 & 38,1 \\
\hline Homens & 49,5 & 32,0 \\
\hline População com idade entre 16 e 24 anos & 52,4 & 30,5 \\
\hline Mulheres com idade entre 16 e 24 anos & 57,1 & 34,5 \\
\hline Homens com idade entre 16 e 24 anos & 47,8 & 25,8 \\
\hline \multicolumn{3}{|l|}{ Classe sócio-econômical } \\
\hline Classe A & 61,1 & 38,3 \\
\hline Classe B & 50,7 & 40,2 \\
\hline Classe C/D & 52,0 & 35,3 \\
\hline Classe E & 47,7 & 19,4 \\
\hline Mulheres pertencentes à Classe $A$ & 61,2 & 46,6 \\
\hline Mulheres pertencentes à Classe B & 45,8 & 37,1 \\
\hline Mulheres pertencentes às Classes C/D & 55,9 & 39,5 \\
\hline Mulheres pertencentes à Classe E & 55,7 & 21,9 \\
\hline Homens pertencentes à Classe A & 60,9 & 32,1 \\
\hline Homens pertencentes à Classe B & 56,7 & 23,9 \\
\hline Homens pertencentes às Classes C/D & 47,5 & 30,5 \\
\hline Homens pertencentes à Classe $\mathrm{E}$ & 42,2 & 17,6 \\
\hline
\end{tabular}

Fonte: Pesquisa Nacional sobre Comportamento Sexual da População Brasileira e Percepções do HIV/AIDS (Secretaria de Assistência à Saúde, Ministério da Saúde).

1 Critério Brasil de Classificação Econômica.

274 pontos ou mais na escala de conhecimento sobre HIV/AIDS.

390 pontos ou mais na escala de conhecimento sobre HIV/AIDS. 
tida, o último grupo, com pouco mais de um quarto da população ou 15,8 milhões de pessoas, apresentou maioria feminina $(56,2 \%)$; idade média de 32 anos, com aproximadamente $31 \%$ dos seus componentes com menos de 25 anos; e cerca de $58 \%$ casados ou unidos. Os dois agrupamentos intermediários, englobando cerca de $50 \%$ da população, apresentaram praticamente a mesma distribuição por sexo observada para o total da população, diferindo quanto à faixa etária: enquanto o grupo 2 apre- sentou um perfil parecido ao último grupo, o grupo 3 se caracterizou pela presença de pessoas mais velhas assemelhando-se ao primeiro agrupamento (Tabela 3 ).

A Tabela 3 mostra ainda que os grupos $1 \mathrm{e}$ 3 se caracterizam por uma menor escolaridade de seus membros, respectivamente $63,9 \%$ e $56,5 \%$ não concluíram o ensino fundamental, isto é, possuem menos de 8 anos de escolaridade. Os grupos 2 e 4 apresentam menos de $50 \%$ das pessoas nessas condições, respectiva-

Tabela 3

Distribuição das pessoas de 16 a 65 anos, por grupos de conhecimento sobre HIV/AIDS e percepção de risco, segundo características demográficas e sócio-econômicas. Brasil, 1998.

\begin{tabular}{|c|c|c|c|c|c|}
\hline \multirow{2}{*}{$\begin{array}{l}\text { Características demográficas } \\
\text { e sócio-econômicas }\end{array}$} & \multicolumn{4}{|c|}{ Conhecimento e percepção de risco (\%) } & \multirow[b]{2}{*}{ Total } \\
\hline & Grupo 1 & Grupo 2 & Grupo 3 & Grupo 4 & \\
\hline Total & 23,3 & 25,0 & 25,2 & 26,5 & 100,0 \\
\hline Base (em 1.000 pessoas) & 13.803 & 14.815 & 14.956 & 15.769 & 59.343 \\
\hline Sexo & 100,0 & 100,0 & 100,0 & 100,0 & 100,0 \\
\hline Masculino & 52,8 & 47,6 & 47,8 & 43,8 & 47,9 \\
\hline Feminino & 47,2 & 52,4 & 52,2 & 56,2 & 52,1 \\
\hline Idade (anos) & 100,0 & 100,0 & 100,0 & 100,0 & 100,0 \\
\hline $16-24$ & 21,8 & 32,3 & 24,8 & 30,7 & 27,6 \\
\hline $25-35$ & 22,1 & 36,1 & 25,0 & 31,5 & 28,8 \\
\hline $36-45$ & 19,1 & 18,1 & 26,2 & 24,4 & 22,1 \\
\hline $46-55$ & 18,6 & 8,1 & 15,0 & 10,6 & 12,9 \\
\hline 56 e mais & 18,4 & 5,4 & 9,0 & 2,8 & 8,6 \\
\hline Idade média (anos) & 39 & 31 & 36 & 32 & 35 \\
\hline Idade mediana (anos) & 38 & 29 & 36 & 32 & 34 \\
\hline Estado conjugal & 100,0 & 100,0 & 100,0 & 100,0 & 100,0 \\
\hline Solteiro & 22,1 & 43,5 & 34,2 & 37,0 & 34,4 \\
\hline Casado(a) ou Unido(a) & 63,7 & 50,1 & 58,1 & 57,6 & 57,3 \\
\hline Viúvo(a) & 8,0 & 1,9 & 4,5 & 0,6 & 3,6 \\
\hline Separado(a)1 & 6,2 & 4,5 & 3,2 & 4,8 & 4,7 \\
\hline Grau de instrução & 100,0 & 100,0 & 100,0 & 100,0 & 100,0 \\
\hline Fundamental incompleto & 63,9 & 39,9 & 56,5 & 48,6 & 52,0 \\
\hline Fundamental completo & 17,4 & 26,7 & 24,1 & 19,3 & 21,9 \\
\hline Médio completo & 15,8 & 26,1 & 14,9 & 27,0 & 21,1 \\
\hline Superior & 2,9 & 7,3 & 4,5 & 5,1 & 5,0 \\
\hline Classe sócio-econômica 2 & 100,0 & 100,0 & 100,0 & 100,0 & 100,0 \\
\hline A & 1,6 & 5,1 & 2,9 & 7,1 & 4,2 \\
\hline B & 18,1 & 25,3 & 16,9 & 24,7 & 21,4 \\
\hline$C / D$ & 68,9 & 63,0 & 69,7 & 63,3 & 66,2 \\
\hline $\mathrm{E}$ & 11,4 & 6,6 & 10,5 & 4,9 & 8,2 \\
\hline
\end{tabular}

Fonte: Pesquisa Nacional sobre Comportamento Sexual da População Brasileira e Percepções do HIV/AIDS (Secretaria de Assistência à Saúde, Ministério da Saúde).

1 Inclui as pessoas que se declararam separadas, desquitadas ou divorciadas.

2 Critério Brasil de Classificação Econômica.

Grupo 1: sem informação e sem percepção de risco; Grupo 2: sem informação e com alguma percepção de risco;

Grupo 3: com informação e sem percepção de risco; Grupo 4: com informação e com alguma percepção de risco. 
mente, 39,9\% e 48,6\%. Em relação à situação sócio-econômica, a presença de pessoas pertencentes à classe A é praticamente inexistente nos grupos 1 e 3, com um décimo de seus componentes pertencentes à classe E. Nos grupos 2 e 4, o percentual de pessoas pertencentes à classe A é superior ao observado na população, aproximadamente um terço de seus componentes pertence às classes $\mathrm{A} \mathrm{e} \mathrm{B}$.

O grupo 1 apresentou o maior percentual de pessoas não sexualmente ativas $(27,4 \%)$. Em todos os grupos, a maioria das pessoas sexualmente ativas estava envolvida em relações estáveis, porém os grupos 2 e 4 apresentaram os maiores percentuais de indivíduos com relações estáveis e eventuais, especialmente o grupo 2 com 19,5\% de pessoas nesta situação. Nesses dois grupos também foi maior a presença de pessoas que tinham apenas relações eventuais (Tabela 4).
Quanto ao uso do preservativo, cerca de 50\% do grupo 1 nunca o utilizou, e dos que já usaram $84,9 \%$ não o utiliza atualmente. O grupo 2 apresentou os maiores índices de uso, 75,9\% já usou e $31,0 \%$ ainda o utiliza, número próximo ao observado para o quarto grupo. O grupo 3 se manteve em uma posição intermediária. $\mathrm{O}$ percentual de pessoas que conheciam locais onde é possível comprar preservativos foi superior a $90 \%$ nos grupos 2 , 3 e 4 , mas no grupo 1 este percentual foi de $86,5 \%$ (Tabela 4 ).

A maioria das pessoas pertencentes ao grupo 1 não falou nenhuma vez sobre AIDS no último mês, no grupo 3 cerca da metade estava nesta situação, enquanto nos grupos 2 e 4 este percentual era pouco mais de $40 \%$. Em todos os quatro grupos a maioria declarou não conhecer pessoalmente um portador do vírus HIV, tendo o grupo 2 apresentado o maior percentual de respostas positivas, $30 \%$ (Tabela 5).

Tabela 4

Distribuição das pessoas de 16 a 65 anos, por grupos de conhecimento sobre HIV/AIDS e percepção de risco, segundo tipo de relação e uso do preservativo. Brasil, 1998.

\begin{tabular}{|c|c|c|c|c|c|}
\hline \multirow{2}{*}{$\begin{array}{l}\text { Tipo de relação e uso } \\
\text { do preservativo }\end{array}$} & \multicolumn{4}{|c|}{ Conhecimento e percepção de risco (\%) } & \multirow[b]{2}{*}{ Total } \\
\hline & Grupo 1 & Grupo 2 & Grupo 3 & Grupo 4 & \\
\hline Tipo de relação & 100,0 & 100,0 & 100,0 & 100,0 & 100,0 \\
\hline $\mathrm{N}$ ão sexualmente ativo 1 & 27,4 & 15,8 & 23,6 & 17,0 & 20,8 \\
\hline Com relação estável & 67,2 & 58,7 & 66,5 & 66,6 & 64,7 \\
\hline Com relações eventuais & 2,7 & 6,0 & 3,8 & 6,0 & 4,7 \\
\hline Com relação estável e eventual & 2,7 & 19,5 & 6,1 & 10,4 & 9,8 \\
\hline Base (em 1.000 pessoas) & 13.803 & 14.815 & 14.956 & 15.769 & 59.343 \\
\hline Uso do preservativo & 100,0 & 100,0 & 100,0 & 100,0 & 100,0 \\
\hline Já usou & 49,8 & 75,9 & 58,6 & 68,3 & 64,0 \\
\hline Nunca usou & 50,2 & 24,1 & 41,4 & 31,7 & 36,0 \\
\hline Base (em 1.000 pessoas) & 10.335 & 12.971 & 11.846 & 13.561 & 48.713 \\
\hline Uso do preservativo atualmente 2 & 100,0 & 100,0 & 100,0 & 100,0 & 100,0 \\
\hline Sim & 15,1 & 31,0 & 18,8 & 28,0 & 23,8 \\
\hline Não & 84,9 & 69,0 & 81,2 & 72,0 & 76,2 \\
\hline Base (em 1.000 pessoas) & 9.969 & 12.464 & 11.399 & 13.028 & 46.860 \\
\hline Locais onde se compra preservativo & 100,0 & 100,0 & 100,0 & 100,0 & 100,0 \\
\hline Informou pelo menos um & 86,5 & 97,1 & 93,4 & 97,5 & 93,8 \\
\hline Não sabia informar & 13,1 & 2,8 & 6,4 & 2,5 & 6,0 \\
\hline Não quis responder à questão & 0,4 & 0,1 & 0,2 & 0,0 & 0,2 \\
\hline Base (em 1.000 pessoas) & 13.803 & 14.815 & 14.956 & 15.769 & 59.343 \\
\hline
\end{tabular}

Fonte: Pesquisa Nacional sobre Comportamento Sexual da População Brasileira e Percepções do HIV/AIDS (Secretaria de Assistência à Saúde, Ministério da Saúde).

1 Inclui os indivíduos que nunca tiveram relações sexuais ou estão sem ter há pelo menos 12 meses.

2 Inclui apenas os indivíduos classificados como tendo algum tipo de relação: estável e/ou eventual.

Grupo 1: sem informação e sem percepção de risco; Grupo 2: sem informação e com alguma percepção de risco:

Grupo 3: com informação e sem percepção de risco; Grupo 4: com informação e com alguma percepção de risco. 
Distribuição das pessoas de 16 a 65 anos, por grupos de conhecimento sobre HIV/AIDS e percepção de risco, segundo a presença da AIDS na vida das pessoas. Brasil, 1998.

\begin{tabular}{lrrrrr}
\hline \multirow{2}{*}{ AIDS no cotidiano } & \multicolumn{5}{c}{ Conhecimento e percepção de risco (\%) } \\
& Grupo 1 & Grupo 2 & Grupo 3 & Grupo 4 & Total \\
\hline Base (em 1.000 pessoas) & 13.803 & 14.815 & 14.956 & 15.769 & 59.343 \\
Número de vezes que falou & 100,0 & 100,0 & 100,0 & 100,0 & 100,0 \\
de AIDS no último mês & & & & & \\
$\quad$ Nenhuma vez & 56,8 & 43,9 & 51,2 & 42,5 & 48,3 \\
$\quad$ Algumas vezes & 28,5 & 38,0 & 39,7 & 38,2 & 36,3 \\
Muitas vezes & 14,1 & 18,0 & 9,0 & 18,1 & 14,9 \\
Não informou1 & 0,6 & 0,1 & 0,1 & 1,2 & 0,5 \\
Conhece pessoalmente & 100,0 & 100,0 & 100,0 & 100,0 & 100,0 \\
algum portador do HIV & & & & & \\
Sim & 21,2 & 31,0 & 17,6 & 24,9 & 23,7 \\
Não & 78,3 & 68,9 & 82,2 & 74,5 & 75,9 \\
Não informou1 & 0,5 & 0,1 & 0,2 & 0,6 & 0,4 \\
\hline
\end{tabular}

Fonte: Pesquisa Nacional sobre Comportamento Sexual da População Brasileira e Percepções do HIV/AIDS

(Secretaria de Assistência à Saúde, Ministério da Saúde).

1 Inclui os indivíduos que não quiseram responder à questão ou não souberam respondê-la.

Grupo 1: sem informação e sem percepção de risco; Grupo 2: sem informação e com alguma percepção de risco;

Grupo 3: com informação e sem percepção de risco; Grupo 4: com informação e com alguma percepção de risco

\section{Discussão e conclusão}

Com base na metodologia adotada mensurouse em pouco mais da metade a parcela da população brasileira que estava minimamente informada a respeito das formas de transmissão do HIV no ano da pesquisa. Como era de se esperar, o nível de informação aumenta conforme ocorre uma melhoria nas condições sócio-econômicas: a diferença entre o percentual de pessoas com um bom nível de informação é de quase 20 pontos entre as classes A e E. As mulheres se mostraram mais bem-informadas que os homens, inclusive entre os jovens de 16 a 24 anos e entre diferentes classes sócio-econômicas. Quanto aos jovens, eles não se mostraram mais bem-informados do que a população em geral.

Esses resultados estão de acordo com os encontrados em outras pesquisas quantitativas. Peruga \& Celentano (1993), comparando 80 estudos desse tipo, realizados em diversos países ocidentais, mostrou que havia associação entre escolaridade, renda e conhecimento sobre o HIV/AIDS. Quanto ao sexo, alguns estudos, na época, já mostravam que as mulheres eram mais bem-informadas em relação ao HIV/AIDS.

Quanto aos subgrupos populacionais, temse que, $23 \%$ da população investigada pertence ao grupo 1 (sem informação e sem percepção de risco) ou seja, vulneráveis ao HIV devido à sua condição de não compreender seu próprio risco de exposição à AIDS. O perfil sócio-econômico desse grupo sugere ainda que, é um grupo vulnerável não apenas aos aspectos relacionados à compreensão de seu próprio risco de contaminação, mas também, ou principalmente, à pobreza. Já o grupo 4, composto de $26,5 \%$ da população, que engloba os indivíduos mais bem-informados e com alguma percepção do próprio risco, apresenta maioria feminina e melhores indicadores sócio-econômicos do que os três restantes, com relativamente mais jovens entre seus componentes do que o primeiro grupo.

As distintas características sócio-econômicas e demográficas observadas nesses dois grupos estão de acordo com Parker \& Camargo Jr. (2000), quando estes autores afirmam que as desigualdades sociais, econômicas e demográficas existentes no Brasil, conformam populações diferentes com distintas probabilidades de infectar-se pelo HIV.

Da mesma forma, quando Fonseca et al. (2000) mostram o declínio, entre 1986 e 1996 , da participação proporcional dos casos de AIDS entre pessoas com maior escolaridade, constatando ainda, que nos casos das mulheres infectadas, observa-se elevadas taxas de incidência de AIDS para a categoria de mais baixa instrução. 
Os grupos 2 e 4, compostos por pessoas que possuem alguma percepção de risco, apresentam muitas características semelhantes, o mesmo ocorrendo entre os agrupamentos 3 e 4, sendo estes caracterizados pela falta de percepção. Tal fato sugere que, mais do que a informação, a percepção é o diferenciador entre a adoção ou não de comportamentos protetores.

Os maiores percentuais nesses grupos de pessoas que conhecem pessoalmente portadores do HIV, e a presença de pessoas com relações esporádicas ou eventuais confirmam que, entre outros fatores, a percepção surge das ex-

\section{Referências}

ANDRADE, D. F.; TAVARES, H. R. \& VALLE, R. C., 2000. Teoria da Resposta ao Item: Conceitos e Aplicações. Caxambu: Associação Brasileira de Estatística.

ANDRÉ, L. M., 1999. Representações e Práticas Preventivas da AIDS em Coletores de Lixo no Município de São Paulo. Tese de Doutorado, São Paulo: Faculdade de Saúde Pública, Universidade de São Paulo.

AYRES, J. R. C. M.; FRANÇA, I. J.; CALAZANS, G. J. \& SALETTI, H. C. F., 1999. Vulnerabilidade e prevenção em tempos de AIDS. In: Sexualidade pelo Avesso: Direitos, Identidades e Poder (R. M. Barbosa \& R. Parker, org.), pp. 49-72, São Paulo: Editora 34/Instituto de Medicina Social, Universidade do Estado do Rio de Janeiro.

BAJOS, N.; DUCOT, B.; SPENCER, B.; SPIRA, A. \& ACSP GROUP, 1997. Sexual risk-taking, socio-sexual biographies and sexual interaction: Elements of the French national survey on sexual behavior. Social Science and Medicine, 44:25-40.

BASTOS, F. I. \& SZWARCWALD, C. L., 2000. AIDS e pauperização: Principais conceitos e evidências empíricas. Cadernos de Saúde Pública, 16(Sup. 1): 65-76.

BRITO, A. M.; CASTLHO, E. A. \& SZWARCWALD, C. L., 2000. AIDS e infecção pelo HIV no Brasil: Uma epidemia multifacetada. Revista da Sociedade Brasileira de Medicina Tropical, 32:207-217.

CARR, A., 1991. Behavior Change in Response to the HIV Epidemic: Some Analogies and Lessons from the Experience of the Gay Communities. Issues Paper 7. HIV and Development Programme. 5 April 2001 <http://www.undp.org,/hiv/publications/ issues/english/issue07e.htm>. periências vividas pelas pessoas. Esses achados são amplamente conhecidos e discutidos na literatura (Bajos et al., 1997; Carr, 1991; McGrath et al., 1993; Paicheler, 1999).

Por fim é importante destacar que o tipo de dados utilizados no estudo possui os limites inerentes a uma pesquisa quantitativa. Apesar disso, a pesquisa domiciliar por amostragem é um poderoso instrumento para o levantamento de informações nas mais diversas áreas do conhecimento, e um importante meio para se testar hipóteses aventadas em pesquisas qualitativas.
CEBRAP (Centro Brasileiro de Análise e Planejamento), 1999. Comportamento Sexual da População Brasileira e Percepções do HIVIAIDS. Relatório Técnico. São Paulo: Ministério da Saúde.

FONSECA, M. G.; BASTOS, F. I.; DERRICO, M.; ANDRADE, C. L. T.; TRAVASSOS, C. \& SZWARCWALD, C. L., 2000. AIDS e grau de escolaridade no Brasil: Evolução temporal de 1986 a 1996. Cadernos de Saúde Pública, 16(Sup. 1):77-87.

McGRATH, J. W.; RWABUKWALI, C. B.; SCHUMANN, D. A.; MARKS, J. P.; NAKAYIWA, S.; NAMANDE, B.; NAKYOBE, L. \& MUKASA, R., 1993. Anthropology and AIDS: The cultural context of sexual risk behavior among urban baganda women in Kampala, Uganda. Social Science and Medicine, 36: 429-439.

MEC (Ministério da Educação), 1999. Saeb 97 - Primeiros Resultados. Brasília: Instituto Nacional de Estudos e Pesquisas Educacionais.

MISLEVY, R. J. \& BOCK, R. D., 1990. Item Analysis and Test Scoring With Binary Logistic Models. Mooresville: Scientific Software, Inc.

PAICHELER, G., 1999. General populations and HIV prevention strategies: From risk to action. Cadernos de Saúde Pública, 15(Sup. 2):93-105.

PARKER, R. \& CAMARGO Jr., K. R., 2000. Pobreza e HIV/AIDS: Aspectos antropológicos e sociológicos. Cadernos de Saúde Pública, 16(Sup. 1):89-102.

PERUGA, A. \& CELENTANO, D. D., 1993. Correlates of AIDS knowledge in samples of the general population. Social Science and Medicine, 36:509-524.

Recebido em 30 de maio de 2003

Versão final reapresentada em 29 de julho de 2003

Aprovado em 29 de agosto de 2003 